\title{
Status of the TransiEnt Library: Transient Simulation of Complex Integrated Energy Systems
}

\author{
Anne Senkel $^{1}$ Carsten Bode ${ }^{1}$ Jan-Peter Heckel ${ }^{2}$ Oliver Schülting ${ }^{3}$ \\ Gerhard Schmitz $^{1}$ Christian Becker ${ }^{2}$ Alfons Kather ${ }^{3}$
}

Hamburg University of Technology, Am Schwarzenberg-Campus 1, Hamburg, Germany

${ }^{1}$ Institute of Engineering Thermodynamics, \{anne.senkel, c.bode, schmitz\}@ tuhh.de

${ }^{2}$ Institute of Electrical Power and Energy Technology, \{jan.heckel, c.becker\}@tuhh.de

${ }^{3}$ Institute of Energy Systems, \{oliver.schuelting, kather\}@ @uhh.de

\begin{abstract}
The open-source Modelica library TransiEnt Library was developed within the research project TransiEnt.EE. This paper presents two major library extensions which were developed in the follow-up research project ResiliEntEE. Modeling of the power sector on transmission grid level is now possible due to the implementation of the complex bus voltage. In the gas sector, the efficiency of mass and energy balance computation was improved. Furthermore, an efficient physical pressure loss model was added leading to more realistic results and faster simulations. One possible application of the library is presented in an exemplary simulation of an integrated energy system. It is shown that the dynamic simulation allows the representation of disturbances and their possible consequences in coupled sectors. Thus, next to cost and $\mathrm{CO}_{2}$ emissions, an integrated energy system can be assessed in terms of its resilience as well.

Keywords: Integrated Energy Systems, Dynamic Interactions, Electricity, Gas, Heat, TransiEnt Library
\end{abstract}

\section{Introduction}

The energy sector is facing severe changes, not only in the upcoming years but already today. In its recent study, Agora Energiewende identifies ten megatrends that will influence the development of the future energy system (Agora Energiewende 2019). Among them are:

- Decarbonization,

- Cost regression for renewables,

- Digitalization,

- Electrification,

- Urbanization,

- Decentralization, and

- Interdependence.

Each of these tendencies will not only lead to a more sustainable but also more complex and dynamic integrated energy system (IES). In Figure 1, typical components of an IES are depicted for the sectors gas, heat, and power. Researchers and decisionmakers alike are facing the challenge of identifying different development paths and their advantages and disadvantages. To be able to analyze and evaluate these, corresponding models need to be developed and the complex and dynamic behavior of the considered IES must be implemented. In terms of evaluation, it is notable that not only cost and $\mathrm{CO}_{2}$ emissions are of interest but resilience is gaining importance as well (Linkov and PalmaOliveira 2017).

In this context, Modelica offers support for multi-domain dynamic simulation and, thus, the simulation of different energy sectors within a single, coherent model. Therefore, multidisciplinary approaches are supported, as are necessary to develop sophisticated models for each sector. Moreover, the object-orientation allows the development of libraries with userfriendly, scalable models that offer a high degree of reusability and adaption.

Based on these considerations, the TransiEnt Library has been developed in the TransiEnt.EE and ResiliEntEE projects (Hamburg University of Technology 2020). Using it, users may implement dynamic interactions in their energy system models and use the results for further assessments.

After a brief presentation of the projects TransiEnt.EE and ResiliEntEE as well as their major outcome, the TransiEnt Library, this paper presents the main extensions of the library in the gas and power sector which were realized in the ResiliEntEE project. To provide insights into the possibilities of the TransiEnt Library, an exemplary model of an IES is presented and its simulation results are discussed.

\subsection{TransiEnt.EE}

The TransiEnt.EE research project was conducted at the Hamburg University of Technology from 2013 to 2017 (Andresen et al. 2017). Its main goal was to identify innovative and reliable ways to efficiently integrate renewable energies into existing urban energy systems to maximize the energy system's self-sufficiency. For their analysis, the researchers focused on the city of Hamburg's energy supply system. In this context, the TransiEnt Library was developed to not only allow the modeling necessary for the concrete research project but also for future studies and related research interests (Andresen et al. 2015).

In TransiEnt.EE, the considered energy systems were analyzed according to their cost and $\mathrm{CO}_{2}$ emissions, enabling efficiency assessments of the considered scenarios with respect to the goals of the German energy transition. 


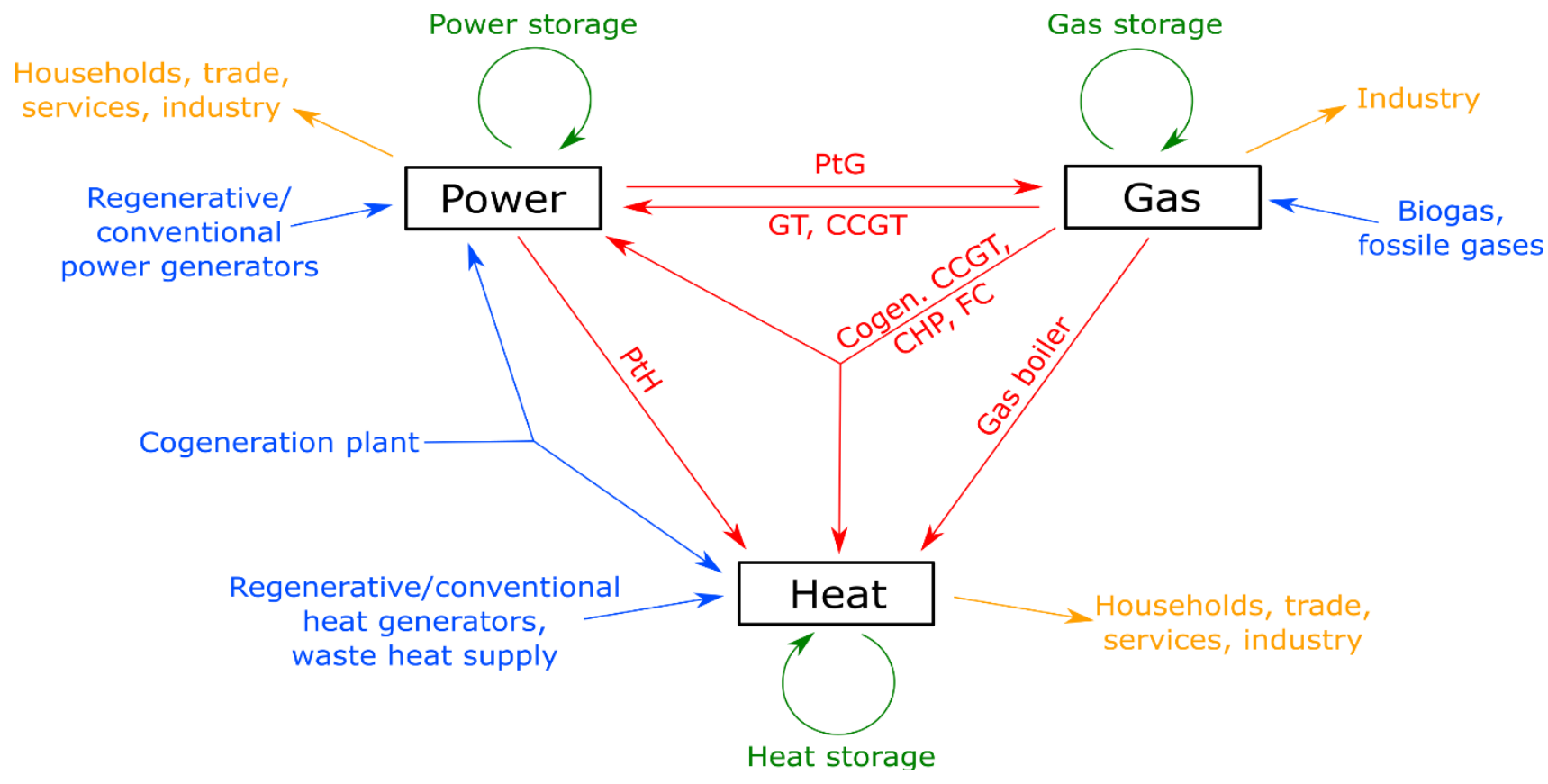

Figure 1. Presentation of the three Sectors (black) with Energy Producers (blue), Conversion Technologies (red), Storages (green) and Consumers (yellow) (PtG: Power-to-Gas, GT: Gas Turbine, CCGT: Combined Cycle Gas Turbine, Cogen. CCGT: Cogeneration Combined Cycle Gas Turbine, CHP: Combined Heat and Power, FC: Fuel Cells, PtH: Power-to-Heat

\subsection{ResiliEntEE}

ResiliEntEE is the follow-up project to TransiEnt.EE conducted at the Hamburg University of Technology from 2017 until 2021. The project used the TransiEnt Library and aimed at refining it twofold:

First, the considered system was enlarged from the city of Hamburg to Northern Germany (including the federal states of Bremen, Hamburg, Lower Saxony, Mecklenburg-Western Pomerania, and Schleswig-Holstein). Towards this, a numerically efficient modeling approach needed to be found to enable fast model generation of the region considering different levels of aggregation and detail. Additionally, the gas and the electric grid modeling needed to be expanded to this superregional scale. Finally, a suitable control for the overall system was designed including the operational planning of renewable power producers, energy conversion technologies, and storage plants as well as the provision of system services and reactions to disruptions.

The second project aim was to use the TransiEnt Library models to evaluate the resilience of the energy system and its subsystems. Therefore, the existing models needed to be refined to show the behavior of the considered systems before, during, and after disturbances. For this purpose, a special focus was laid on the electric grid, expanding the models to spatially resolved, stationary and dynamic computations (Heckel and Becker 2019). Furthermore, the connections between the sectors were revised to be able to answer the questions of how disruptions in one sector affect other sectors.

Finally, a quantitative evaluation method was developed to assess the resilience of energy systems using dynamic simulation results (Senkel, Bode, and Schmitz 2021, 2019). This enables the determination of the resilience of future energy supply systems against stressors such as component failure, weather extrema, control errors, and others. This enables the comparison of different possibilities towards improving system resilience.

\subsection{The TransiEnt Library}

The TransiEnt Library provides models for typical energy system components, including energy producers, consumers, storage units, conversion plants, and grid components (Hamburg University of Technology 2020). The resulting library structure is depicted in Figure 2. A detailed introduction to a preliminary version of the TransiEnt Library is given by Andresen et al. (2015).

Using the TransiEnt Library, the fluctuating energy provision of renewable energies can be modeled dynamically, allowing investigations into their influence on the energy system. Due to the dynamic simulation, dynamic interactions between the sectors and their impact on the flexibility and stability of the overall system can be depicted. This allows sensitivity analyses and the evaluation of different integration and control strategies. Owed to the multi-domain approach of Modelica, this can be done in one model covering all sectors, allowing a detailed analysis of the overall system. One example is the investigation of excess power utilization in the heat sector (Bode and Schmitz 2019).

Towards deduplication, the TransiEnt Library uses components of the ClaRa Library (Hamburg University of Technology, TLK-Thermo GmbH, and XRG Simulation GmbH 2021), the TILMedia Library (TLK-Thermo GmbH and Institut für Thermodynamik, Technische Universität Braunschweig 2021), and the Buildings Library (Wetter et al. 2014).

The ClaRa Library allows the modeling of the transient thermal behavior of power plants and related systems. The TransiEnt Library reuses basic components from that library, including but not limited to pipes, heat exchangers, and valves. Furthermore, certain components of the gas sector are based on 
TransiEnt

\section{(i) UsersGuide}

$>$ Basics

$>$ Components

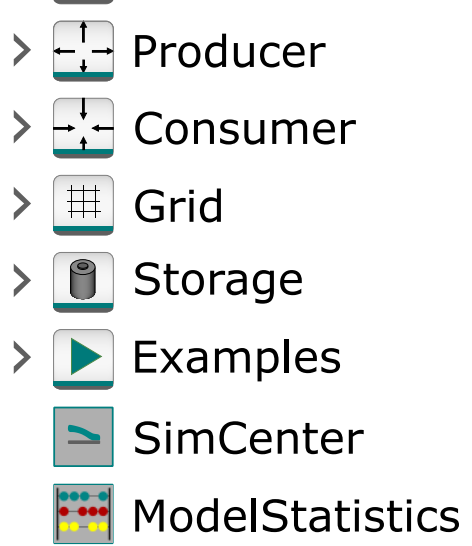

Figure 2. Structure of the TransiEnt Library

models from the ClaRa Library (see section 2.2), for example, pipe and valve models.

The TILMedia Library provides thermophysical properties for various fluids and solids. The TILMedia Library models provide better numerical performance and robustness compared to Modelica.Media (Modelica Association 2020). However, coupling of models using the different media models is possible and a suitable adapter model is included in the TransiEnt Library.

From the Buildings Library, models for the heat transfer within walls and their sky radiation exchange as well as the air volume within a building are used to provide a low-order heat consumer model and thus the modeling of cool-down and heatup processes in buildings connected to the considered energy system.

\section{New Developments and Components}

As a result of the ResiliEntEE project, several new developments and models were implemented in the TransiEnt Library. Especially the gas and power sector modeling were enhanced, as described in the following section. Further developments and results are documented by Bode et al. (2021).

\subsection{Modeling of the Power Sector}

Electric power systems can be modeled on different levels. On the one hand, the focus of dynamic modeling can vary, and, on the other hand, it is possible to differentiate between the levels of modeling the grid connections. In terms of simulation, the simplest option is a steady-state simulation referred to as load flow calculation. In load flow calculations, the grid state is calculated in the form of complex bus voltages based on the representation of the alternating voltages and currents as quasi stationary root mean square (RMS) phasors. The grid state is influenced by the load and generation as well as the switch settings in the electric grid.
To focus on the dynamics, the RMS simulation, also based on phasors, can be used. The frequency of the electric voltage is chosen as an additional variable. This approach allows the use of numerical integrators with variable step sizes above $20 \mathrm{~ms}$ in $50 \mathrm{~Hz}$ systems, enabling efficient simulations of entire years. The RMS simulation approach can assess the electromechanical processes and the behavior of the controllers that ensure the operation of the electric grid.

Lastly, the electric energy system can be simulated using the electromagnetic transient simulation approach. In this approach, voltages and currents are time-dependent including their sinusoidal oscillation. The electromagnetic transient simulations require numerical integrators with step sizes below $20 \mathrm{~ms}$, which have high numerical cost.

In the TransiEnt Library, the RMS approach is chosen. This allows numerically efficient simulations since the stiffness of the problem of the IES simulation can be reduced. Stiff problems occur when time constants from a large range of orders of magnitude are part of the problem and the numerical integrator needs to adapt the step size to the lowest time constant. This is the case for IES simulations when the electric subsystem is simulated using the electromagnetic transient approach. Processes in the heat and gas sector have higher time constants starting at approximately a few seconds, as shown in Figure 3, which gives an overview of time constants of different processes from the power, heat, and gas sector. In addition, the time intervals of the stability phenomena in electric power systems are given. The relevant stability phenomena of the electric subsystem in IES are frequency and voltage stability.

With the RMS simulation approach of the electric energy system, time constants starting at approximately $100 \mathrm{~ms}$ are regarded. The RMS simulation of electric power systems becomes steady state when neither load and generation are changed nor disturbances occur. Using appropriate models for the IES simulation within the RMS approach, problems' stiffness can be reduced. Hence, processes in the IES with time constants, starting at approximately one second, are considered. In contrast, electromagnetic transient simulations always consider time constants of $20 \mathrm{~ms}$ and lower.

The different levels of modeling the grid connections can be demonstrated through the evolution of electrical interfaces in the TransiEnt Library. In the TransiEnt.EE project, the electric grid was modeled as a copper plate with ideal interconnections. This modeling is based on the ActivePowerPort interface which contains the active power as a flow variable and the frequency of the electric grid as a potential variable. With this interface, all electrical components modeled in a simulation model are connected with each other at one bus bar.

In the next step, the modeling was extended to the modeling of radial grid structures as these structures often occur in low voltage grids. For this approach, the ApparentPowerPort was introduced. This interface also considers the electric bus voltage magnitude as a potential variable and the reactive power as a flow variable.

In the ResiliEntEE project, interconnected electric power systems, as they can be found on the transmission grid level, are modeled. Due to this extended scope, a new electrical interface was introduced (Heckel and Becker 2019): The ComplexPowerPort adds the complex bus voltage angle as a potential variable to the modeling. Thus, the complete complex bus voltage is part of the interface leading to the appropriately named ComplexPowerPort. 


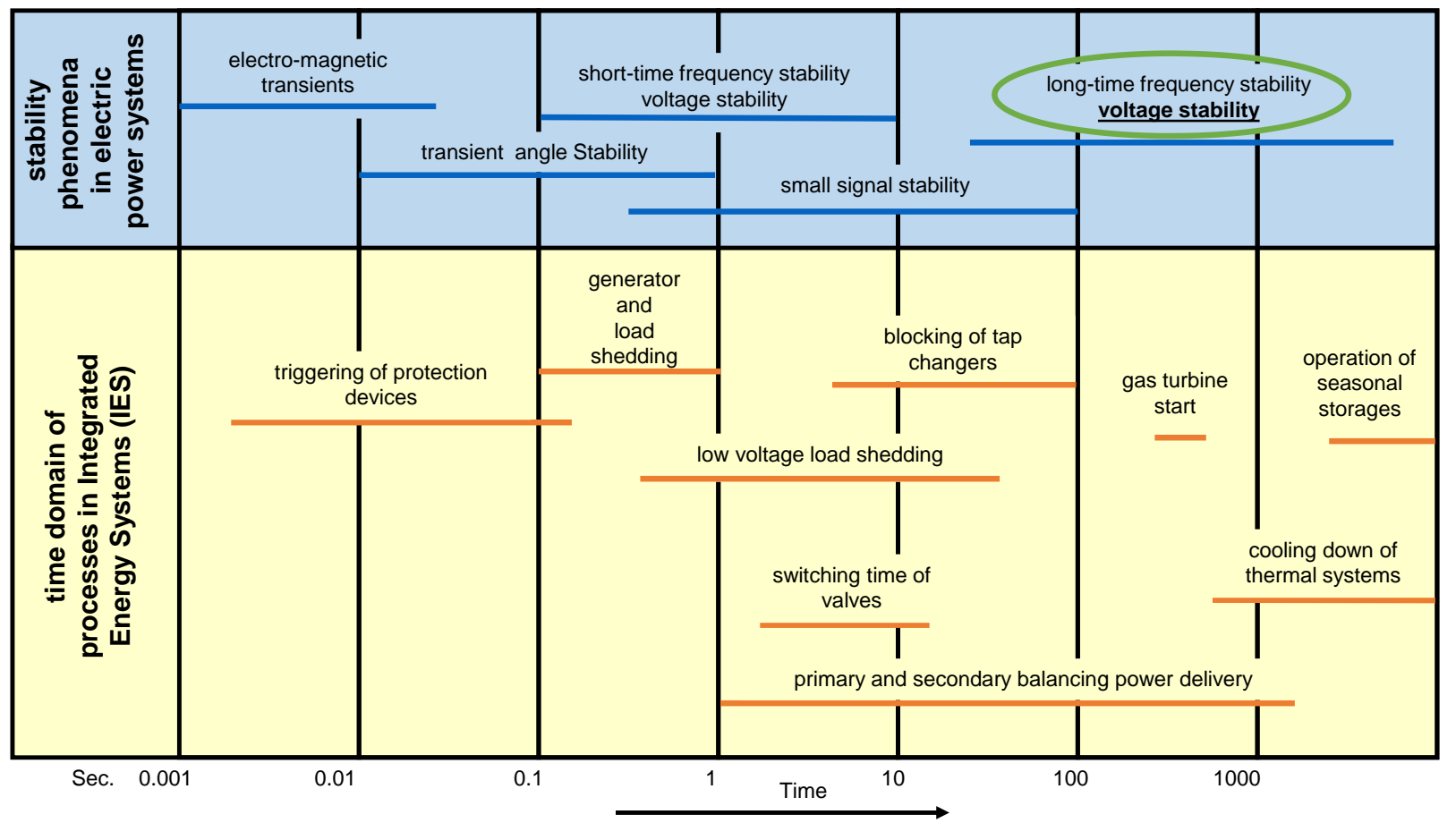

Figure 3. Overview of time constants of processes in IES and the corresponding stability phenomena in electric grids

At two flow variables and three potential variables, the interface is over-determined. The frequency is the additional variable that is part of the modeling due to the RMS simulation approach. For the problem of overdetermined interfaces, the Modelica Specification Section 9.4 gives a workaround (Modelica Association 2017). This workaround is followed here: A custom frequency type with an equalityConstraint is defined for the electric frequency of the grid. With the definition of grid busses as "potential roots" and the grid connection components as "branches" of a "connection tree", this approach allows the RMS modeling of electric power systems with the ComplexPowerPort. All mentioned electrical interfaces in the TransiEnt Library are unipolar allowing flexible modeling in a clearly arranged way.

With the introduction of the ComplexPowerPort, new component models have been added to the TransiEnt Library. These models can be divided into steady-state and dynamic components. As steady-state components, the different bus types, which are considered in the modeling of electric energy systems, are implemented as boundary conditions. Boundary conditions set variables at the interface to given values allowing other variables to take arbitrary values. The bus types are:

- Slack Bus: Voltage magnitude and angle are given; the frequency is given for steady-state calculation and the other quantities are calculated.

- PV Bus: Active power and voltage magnitude are fixed; other quantities are calculated. This bus model is used for power plants with voltage control.

- PQ Bus: Active and reactive power are given; other quantities are calculated. PQ Busses mainly represent loads.

Transmission line models based on the pi network representation in two-port representation model the grid connections. The transmission line model was validated with different commercial and open-source steady-state tools. For the dynamic simulation, these models can be reused because the grid connections are assumed steady-state in the RMS simulation of electric energy systems. Additionally, different transformer models allow the modeling of interconnected and spacious electric transmission systems.

As dynamic component models, new models for synchronous generators have been implemented. The TransiEnt Library models allow the modeling of synchronous machines at different levels of detail. All models regard the electromechanical behavior but differ in the electric sub model, which links the voltage magnitude and the active as well as reactive power.

The models can be split into one- and two-axis models. Oneaxis models are suitable for the description of cylindrical rotor machines. Moreover, the two-axis description, based on the Park Transformation (Park 1929), allows a more general description of non-symmetric electric machines.

The different levels of detail enable the adaption of the modeling to the simulation scope. Thus, the trade-off between high accuracy and affordable simulation costs can be resolved in the context of each problem to be simulated.

In addition to the synchronous machines, different motor models, such as an induction motor, have been added to the TransiEnt Library. These models allow a more detailed consideration of the integration of the power sector with the other sectors of an IES through conversion plants.

\subsection{Modeling in the Gas Sector}

The TransiEnt Library contains all models necessary to simulate future gas grids. An example model is shown in

Figure 4 that depicts how the components can be connected. Required fluid properties are supplied by the TILMedia Library.

The essential components of a gas grid are the pipes transporting the gas. The respective model is based on the ClaRa Library and uses the finite volume method for one-dimensional flow. Each control volume contains an overall mass balance, component mass balances, and an energy balance. The heat 
transfer can be modeled using different heat transfer models inside the pipe and additional wall and external heat transfer models.

To work more efficiently in large networks, different simplifications were added for the model in the TransiEnt Library: It is possible to use isothermal flow, which is a common assumption in gas grids (Cerbe and Lendt 2017) to eliminate the specific enthalpy as a state.

In addition, the model was augmented with simplified component mass balances: If the gas in the grid has a constant composition, the mass fractions are eliminated from the system of equations. Furthermore, if, for example, natural gas with constant composition is transported in the grid and hydrogen is admixed, the hydrogen fraction is the only truly variable component and all others depend on it. In this case, the dependent fractions can be calculated by scaling the nominal composition of the main gas accordingly. This also reduces the number of states.

In the ClaRa Library, all pressure loss models are either linear or quadratic and depend on nominal values. To enable simpler pipe parametrization and gain more realistic results, a pressure loss calculation based on Cerbe and Lendt (2017) is implemented by adapting the equations from Modelica.Fluid.Dissipation (Modelica Association 2020): Pipe discretization can be avoided by using the pressure loss equation for a compressible fluid under the assumption that the quadratic mean pressure equals the arithmetic mean pressure. This leads to a small error but also efficient simulation since the equation does not contain quadratic pressures and each pipe can consist of only one control volume. The required dynamic viscosity is not supplied by the TILMedia Library, so linear correlations based on media data from REFPROP (Huber et al. 2018) for natural gas, methane-hydrogen mixtures, and pure hydrogen are used.

In addition to pipes, junctions are an important part of the gas grid since they enable the mixing of different gas flows. Additionally, they connect pipes and their corresponding pressure losses and volumes in a numerically efficient way. The junction model is also based on a model from the ClaRa Library and consists of a volume with an arbitrary number of gas ports. The volume contains the same equations as the control volume of a pipe with the same simplifications for component mass as well as energy balances. Linear pressure losses between the volume and each gas port can be added to avoid nonlinear systems of equations in certain cases.

To balance gas supply and demand, gas storage units are necessary. The TransiEnt Library contains different storage models which differ in their level of detail. First, the gas has either constant or variable composition. Second, the gas storage either contains a fluid model, with which temperature and pressure in the gas storage can be calculated, or simply uses a constant pressure. The latter is the faster model but neglects the gas state in the storage. The model containing a fluid model is similar to the junction model but different heat transfer models, typical for certain kinds of gas storage, can be used: cavern storage, gas pressure vessel, or adiabatic storage. Furthermore, the pipes, through which the gas enters and leaves the storage, can be included in the model: either using a pressure loss and heat transfer model or neglecting pressure losses and assuming constant outlet temperatures.

Furthermore, suitable boundary conditions are needed to simulate a gas grid. The TransiEnt Library contains several basic boundary conditions that always set three values:

- pressure, mass flow rate, volume flow rate at standard conditions, or enthalpy flow rate

- mass or molar fraction, and

- temperature or specific enthalpy.

Gas consumers also act as boundary conditions for the gas grid. Different kinds of gas-fired heat producers, for example, gas boilers, gas heat pumps, and combined heat and power plants, as well as gas-fired power plants, for example, gas turbines and combined-cycle power plants, are part of the TransiEnt Library.

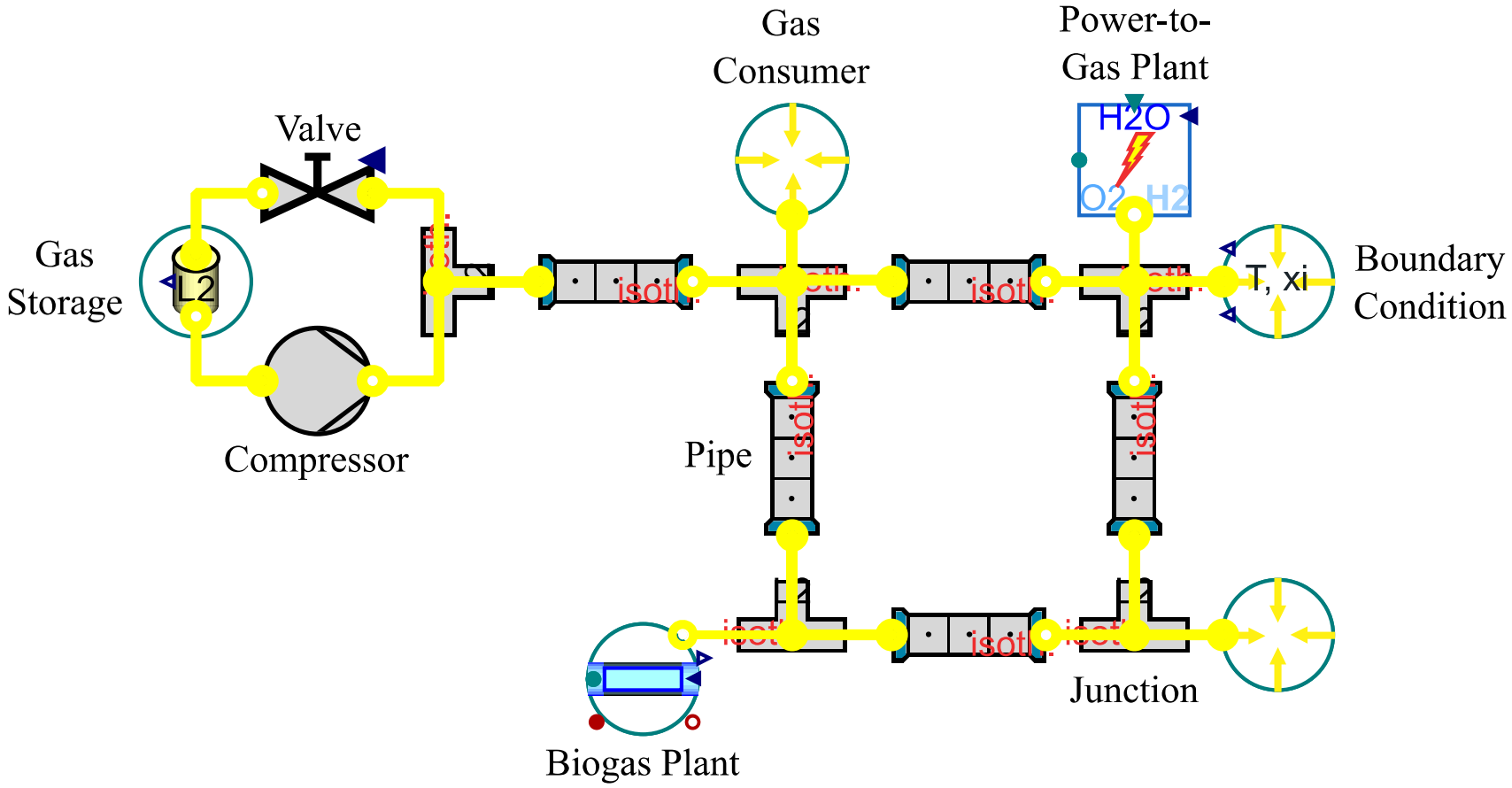

Figure 4. Gas Model with New Components 
Major gas-producing units of the future will be biogas and Power-to-Gas plants. In a biogas plant, different organic substrates are transformed into biogas using bacteria. The model in the TransiEnt Library contains the ADM1 model (Klimiuk et al. 2015), which models the transformation of different organic components. Moreover, correlations for the power demand of different stirrers as well as the heat transfer from the heater to the reactor and from the reactor to the ambient are included.

The second technology, Power-to-Gas, uses electricity to generate hydrogen via electrolysis. The core component, the electrolyzer, is either modeled using an efficiency curve or more detailed physics of the electrolyzer (Webster and Bode 2019). Both models include either quasi-stationary, first-order, or second-order dynamics as well as the possibility to give a certain amount of waste heat to a connected component.

In some Power-to-Gas plants, the hydrogen is transformed to synthetic natural gas using $\mathrm{CO}_{2}$ to be fed directly into the natural gas grid. Different models are included in the TransiEnt Library which either use reaction kinetics, reaction equilibrium, or a constant conversion rate. The required $\mathrm{CO}_{2}$ can be separated from air, modeled by a simple direct air capture plant model.

All parts of the Power-to-Gas plant can be combined to socalled feed-in stations which contain suitable controllers as well as storage units.

Also, the TransiEnt Library contains a model for a steam methane reformer including related reactor models, for example, a pre-reformer and a water-gas shift reactor. Additional minor components, which are part of the TransiEnt Library, are simple compressor, heat exchanger, and valve models.

\section{Example of Use}

The following chapter presents the CoupledLargeScale model from the Example Package of the TransiEnt Library. In this model, the sectors gas, heat, and power are integrated and coupled by a combined-cycle power (CCP) plant with heat extraction and an electrolyzer. The structure of the IES is depicted in Figure 5, the parameters of the system are listed in Table 1.

\subsection{Model Description}

In the gas sector, a gas grid containing one loop supplies gas consumers at three points in the grid (NW, NE, SE). In the northwest, a constant gas demand is modeled as it is often the case for industrial consumers. In the east, the gas is used to supply gas boilers for the heat supply of housing areas. Additionally, the CCP plant in the southeast burns gas to provide heat to a district heating network (DHN) and power to the electric grid. The gas is provided to the gas grid by a gas source in the southwest at a pressure of 12.5 bar. Moreover, the electrolyzer in the southwest uses excess power to produce hydrogen which is also fed into the gas grid.

The demand in the heating sector is modeled using a reducedorder approach considering the thermal capacities and conductances of the houses as well as thermal heat gains and ventilation losses. To achieve a reasonable computation time, the housing areas are modeled by computing the heat demand for one characteristic house and scaling it up accordingly. The heat is transported to the households in two different ways. The housing areas in the eastern part of the gas grid are supplied by gas boilers which are controlled in order to reach the set room temperature.

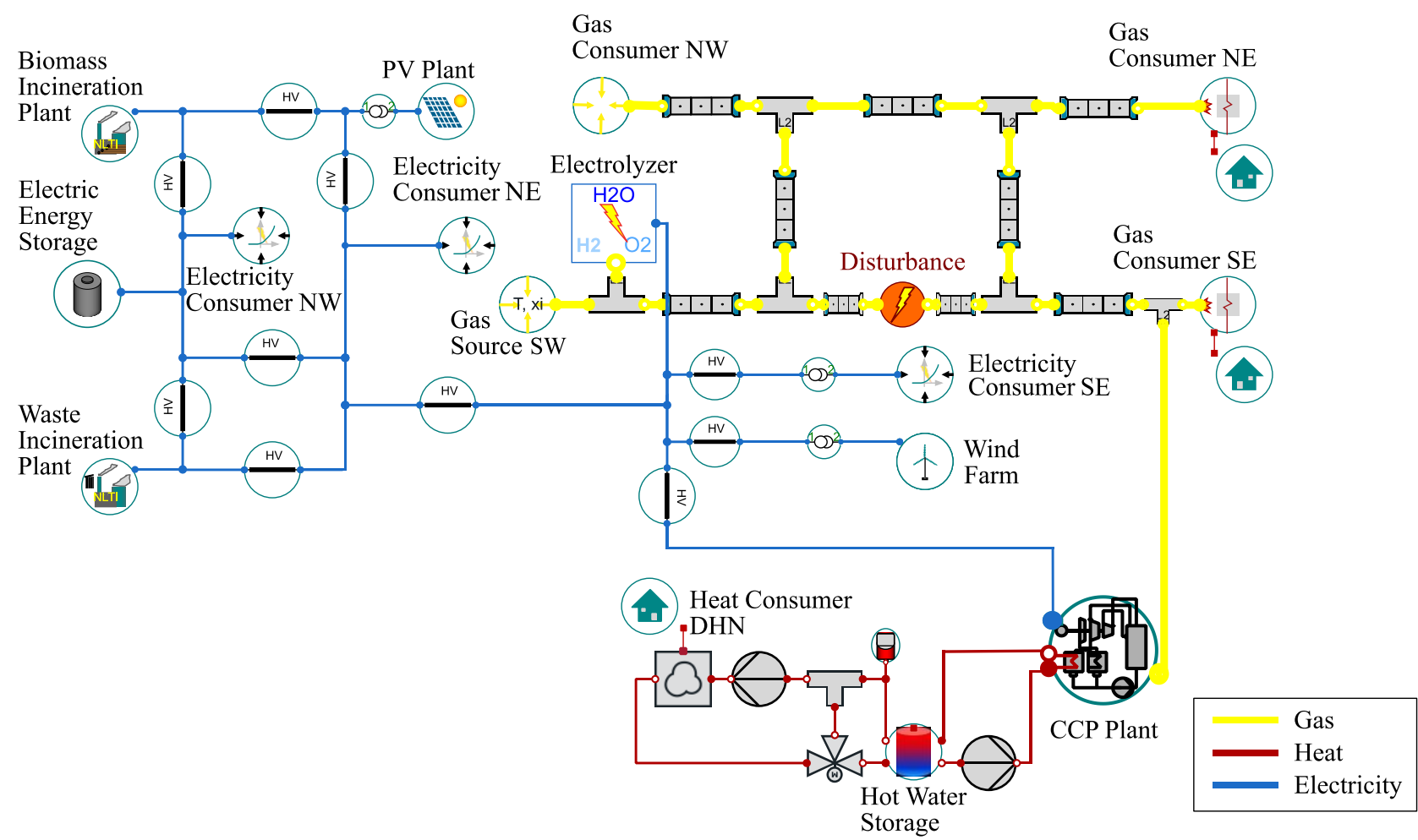

Figure 5. Graphical Laver of the Investigated Integrated Energy System 
Table 1. Parametrization of the Investigated IES

\begin{tabular}{lr}
\hline Parameter & \\
\hline Gas Sector & $12.5 \mathrm{bar}$ \\
\hline Pressure Gas Source & 2 bar \\
Minimum Pressure Gas Boiler & 2 bar \\
Minimum Pressure CCP Plant & $47 \mathrm{~km}$ \\
Total Pipeline Length & $4400 \mathrm{~m}^{3}$ \\
Gas Grid Volume & \\
\hline Heat Sector & 58100 \\
\hline Number of Households NE & 29050 \\
Number of Households SE & 6260 \\
Number of Households DHN & $280 \mathrm{MW}$ \\
Nominal Heat Flow Rate Gas Boiler NE & $140 \mathrm{MW}$ \\
Nominal Heat Flow Rate Gas Boiler SE & $30 \mathrm{MW}$ \\
Nominal Heat Flow Rate CCP Plant & $1720 \mathrm{~m}{ }^{3}$ \\
Buffer Storage Volume DHN & $80{ }^{\circ} \mathrm{C}$ \\
Supply Temperature DHN & $240 \mathrm{~kg} / \mathrm{s}$ \\
Nominal Mass Flow Rate DHN & $140 \mathrm{MW}$ \\
Nominal Power Electric Boiler & 0.95 \\
Efficiency Electric Boiler & \\
\hline Power Sector
\end{tabular}

Power Sector

\begin{tabular}{lr}
\hline Nominal Power Biomass Incineration & $80 \mathrm{MW}$ \\
Plant & \\
Nominal Power Waste Incineration Plant & $100 \mathrm{MW}$ \\
Nominal Power Wind Park & $60 \mathrm{MW}$ \\
Installed PV Plant Area & $500 \mathrm{~m}^{2}$ \\
Nominal Power CCP Plant & $60 \mathrm{MW}$ \\
Nominal Power Electrolyzer & $1 \mathrm{MW}$ \\
Maximum Discharging Power Electric & $50 \mathrm{MW}$ \\
Energy Storage & \\
Maximum Charging Power Electric & $70 \mathrm{MW}$ \\
Energy Storage & $6 \mathrm{GWh}$ \\
Storage Capacity Electric Energy Storage & 2.6 \\
Proportional Load Factor NW & 2.5 \\
Proportional Load Factor NE & 0.8 \\
Proportional Load Factor SE &
\end{tabular}

The households in the south, however, are connected to their heat supply via the heating network. Hence, their heat supply is connected to the operation of the CCP plant which is not only controlled according to the current heat demand but also to the current electricity demand and production. Therefore, a hot water storage is placed between the heat consumer and the CCP plant, decoupling the two from each other. Thus, the heat supply of the consumers is ensured by a) controlling the supply temperature via a backmixing valve according to a heating curve depending on the ambient temperature and b) by controlling the mass flow rate through the heat exchanger at the consumer to reach the set room temperature. The heat output of the CCP plant is determined according to the set temperature of the top volume of the hot water storage and the physical constraints of the combined heat and power generation.

The electricity demand is modeled using SLP load profiles for agriculture, households, and industry (Price et al. 1993). Additionally, the frequency dependency of the consumers is modeled according to Price et al. (1993). The power generation units are biomass and waste incineration plants as well as a PV plant and a wind park, which are dependent on the solar irradiation and the wind velocity, respectively. To avoid curtailment of the PV plant and wind park, excess power is stored in an electric energy storage or used to produce hydrogen in the electrolyzer. The power provision is controlled according to the merit order: PV and wind plants - CCP plant - discharging the electric energy storage - garbage incineration plant biomass incineration plant. For more detailed information including parametrization, refer to Senkel, Bode, and Schmitz (2021).

This energy system is modeled to be disturbed by the closure of the southern gas pipeline for 14 hours at the beginning of an exemplary February. At this time of the year, the lowest ambient temperatures and the lowest feed-in of renewable energy occur and therefore the strongest effects of the disturbances can be observed. The software used is Dymola (Dassault Systèmes 2021) with the Esdirk45a solver and the tolerance $10^{-6}$. In the next section, the simulation results of the simulation with and without the induced disturbance are discussed.

\subsection{Simulation Results}

In Figure 6, the simulation results for the gas pressure, the aggregated enthalpy flow rate, the room temperature, the characteristic heat flow rate, the grid frequency and the electric power of the undisturbed and disturbed IES are depicted. The gas pressure is fluctuating since the gas demand within the gas grid varies as well, especially because of the ambienttemperature-dependent heat demand. It can also be noticed how the gas pressure decreases with increasing distance to the gas source as the pressure losses in the pipelines accumulate. When the gas pipeline in the south is closed, the pressure drops drastically, especially in the southeast since the distance through the gas grid increases the most. In this part of the grid, the controls of the CCP plant and the gas boiler react to this drop by shutting down the affected assets to protect them from too low pressures. Because of that, the gas demand in the grid decreases and the pressure recovers to a level around 8 bar. However, the original level of 10 to $12 \mathrm{bar}$ is not reached until after the disturbance ceases. Here, the CCP plant and gas boilers are turned on with a one-hour time delay resulting in a small peak right after the pipeline is opened.

When looking at the enthalpy flow rate, the temperature dependence of the gas consumers in the east and, thus, their fluctuating gas demand becomes notable. In contrast to this, the gas consumer in the northeast has a constant gas demand. Since the nominal electric power of the CCP plant is twice as high as its maximum heat flow rate, its gas demand is dominated by the current power demand. Hence, its gas demand is constant except for low-load periods at night. Since only the CCP plant and the gas boilers are turned off, they are the only gas consumers for which the gas demand cannot be met during the disturbance. 


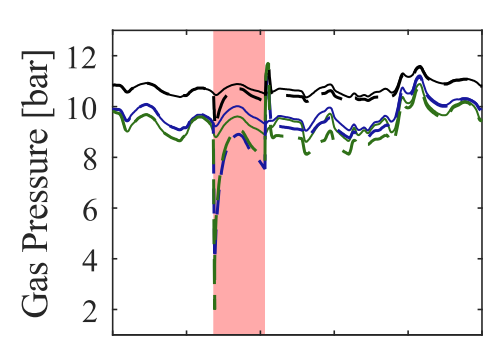

Feb Feb Feb Feb

4th 5th 6 th 7 th

茫
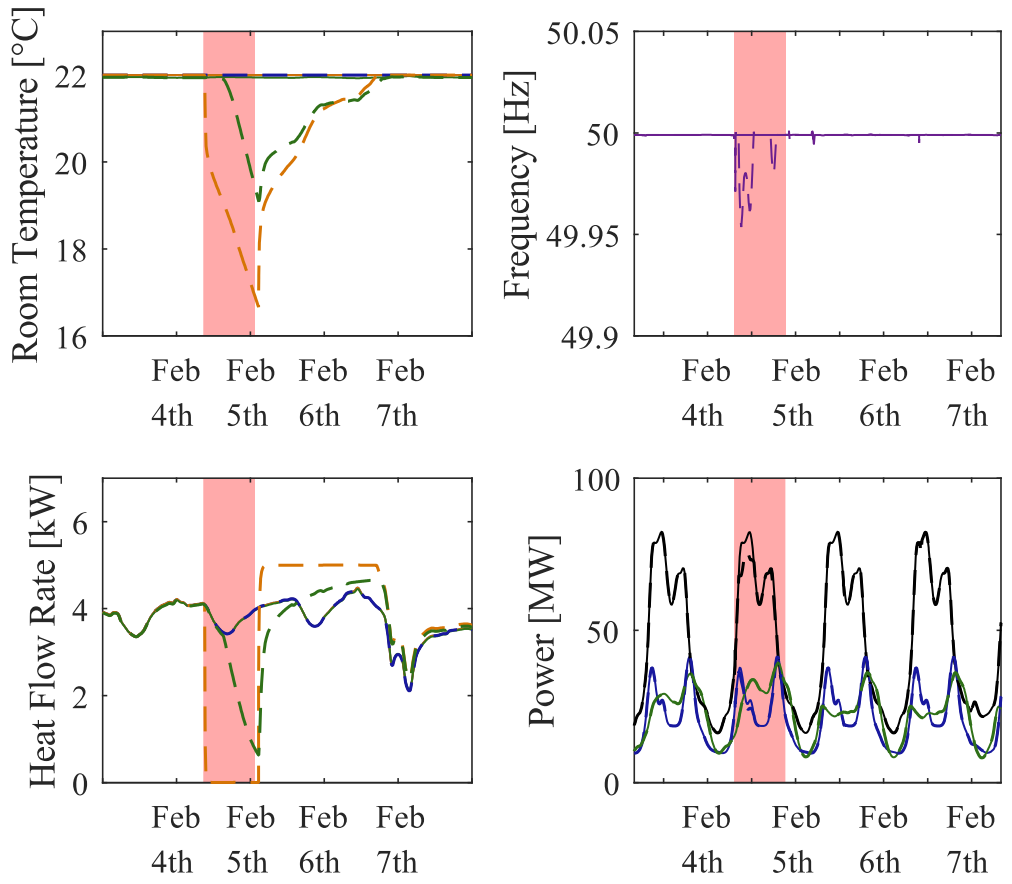

Figure 6. Simulation Results of the Disturbed and Undisturbed IES 
The shutdown of the gas supply for the gas boilers and CCP plant also has consequences for the attached heat consumers. The shutdown of the gas boilers induces an immediate shutdown of the heat supply to the heat consumers in the southeast. Thus, their heat flow rate becomes zero and the houses cool down as seen in the drastic drop of the respective room temperatures.

The households supplied by the heating network do not cool down as fast as the southeastern ones. Here, the hot water storage is able to uphold the heat supply for several hours before it is completely discharged. After that, the heat supply also drops, alongside the room temperature.

After the disturbance, the houses need to be reheated. Therefore, the heat flow rate exceeds the heat demand in the undisturbed case. For the consumers in the southeast, the gas boiler is set to its maximum heat flow rate until the temperature recovers. In the DHN, the heat flow rate to the consumer is controlled differently. Moreover, the thermal inertia of the cooled water in the hot water storage leads to an additional delay in the provision of the maximum heat flow rate.

For the heat consumers in the northeast, the gas and, thus, the heat supply is not affected by the pipeline closure which is why their heat demand and set room temperature can be met at all times.

In the power sector, the other producers react to the shutdown of the CCP plant and the following frequency drop by providing balancing power. However, the demand is still higher than the supply. Hence, the frequency drops and reaches its minimum at $49.95 \mathrm{~Hz}$. In response, the power consumers with the highest proportional load factors (NW and NE) react with an electric load reduction. When the demand becomes smaller, the power supply is sufficient enough to satisfy the demand of all power consumers. At this point, the frequency also returns to its set point of $50 \mathrm{~Hz}$.

The presented simulation results show that a detailed analysis of a disturbed complex system is possible using dynamic simulation. The models of the TransiEnt Library offer detailed modeling and, thus, simulation of the necessary effects. Since this is also true for the energy conversion technologies, the consequences of the disturbance can not only be seen in the directly affected sector but also in the sectors coupled with it. Based on these simulation results, a quantitative assessment of the system's behavior is now possible. Senkel, Bode, and Schmitz (2021) describe a suitable approach and provide more information on the simulation of a system's resilience using Modelica.

\section{$4 \quad$ Summary and Outlook}

In this paper, the status of the TransiEnt Library after the completion of the ResiliEntEE project is presented. Due to the spatial expansion of the region of interest to Northern Germany, the models of the gas and power sector were extended.

In the power sector, the ComplexPowerPort, which integrates the complete complex bus voltage into the modeling, was introduced. This allows the modeling of interconnected electric power systems as they can be found on transmission grid level. In this context, existing models in the TransiEnt Library were revised according to this modeling approach and new models were added.

In the gas sector, the numerical efficiency of the concerning models was improved by simplifying the computation of the mass and energy balances. Furthermore, the pressure loss calculation is accelerated through various improvements. This leads to a strong reduction of numerical effort and enables gas grid calculation within the scope of Northern Germany.

To present the possibilities of the TransiEnt Library, an Integrated Energy System is discussed. With this system, it can be shown how a disturbance in the gas grid affects the integrated heat and power sectors, too. This dynamic simulation of disturbances and their consequences gives the foundation of a quantitative assessment of resilience. In this context, sensitivity analyses and the comparison with system cost and $\mathrm{CO}_{2}$ emissions are possible.

With the completion of the project ResiliEntEE in June of 2021, all developed library extensions are freely available under the terms of the Modelica license agreement. Because of the high interest in the TransiEnt Library, a consortium was founded to promote further development. In this context, the projects IntegraNet and IntegraNet II already use the TransiEnt Library (Benthin et al. 2020). Therefore, the involved institutes Fraunhofer UMSICHT and Gas- and Wärme-Institut Essen e.V. as well as the XRG Simulation $\mathrm{GmbH}$ plan on contributing to the TransiEnt Library in the future.

The current version of the TransiEnt Library can be downloaded using the following link: https://www.tuhh.de/transient-ee/en/.

\section{Acknowledgments}

The authors greatly acknowledge the funding from the German Federal Ministry of Economic Affairs and Energy for the project "ResiliEntEE - Resilienz gekoppelter Energienetze mit hohem Anteil Erneuerbarer Energien" (ResiliEntEE - Resilience of integrated energy networks with a high share of renewable energies, project number: 03ET4048).

\section{References}

Agora Energiewende (2019). "European Energy Transition 2030: The Big Picture. Ten Priorities for the Next European Commission to Meet the EU's 2030 Targets and Accelerate Towards 2050". URL: https://www.agoraenergiewende.de/en/publications/european-energytransition-2030-the-big-picture/ (visited on 2021-02-15).

Andresen, Lisa, Pascal Dubucq, Ricardo Peniche Garcia, Günter Ackermann, Alfons Kather, and Gerhard Schmitz (2015). "Status of the TransiEnt Library: Transient Simulation of Coupled Energy Networks with High Share of Renewable Energy". In: Proceedings Modelica Conference 2015, edited by Modelica Association, 695-705. Linköping Electronic Conference Proceedings: Linköping University Electronic Press. DOI: 10.3384/ecp15118695.

Andresen, Lisa, Pascal Dubucq, Ricardo Peniche Garcia, Günter Ackermann, Alfons Kather, and Gerhard Schmitz (2017). "Transientes Verhalten gekoppelter Energienetze mit hohem Anteil Erneuerbarer Energien, Abschlussbericht des Verbundvorhabens: Laufzeit des Verbundvorhabens: 01.05.2013 bis 30.04.2017". DOI: 10.2314/GBV:1002659345.

Benthin, Jörn, Anne Hagemeier, Annika Heyer, Philipp Huismann, Joachim Krassowski, Christine Settgast, Ben Wortmann, and Klaus Görner (2020). "Gemeinsamer Abschlussbericht des Forschungsvorhabens Integrierte Betrachtung von Strom-, Gas- und Wärmesystemen zur modellbasierten Optimierung des Energieausgleichs- und 
Transportbedarfs innerhalb der deutschen Energienetze". DOI: 10.13140/RG.2.2.17052.44166.

Bode, Carsten, Jan-Peter Heckel, Oliver Schülting, Anne Senkel, Christian Becker, Alfons Kather, and Gerhard Schmitz (2021). "Resilienz gekoppelter Energienetze mit hohem Anteil Erneuerbarer Energien: Abschlussbericht des Verbundvorhabens".

Bode, Carsten, and Gerhard Schmitz (2019). "Influence of Excess Power Utilization in Power-to-Heat Units on an Integrated Energy System with $100 \%$ Renewables". In: Proceedings of the 13th International Modelica Conference, 413-22. Linköping Electronic Conference Proceedings: Linköping University Electronic Press. DOI: 10.3384/ecp19157413.

Cerbe, Günter, and Benno Lendt, eds. (2017). Grundlagen der Gastechnik: Gasbeschaffung - Gasverteilung Gasverwendung. With the assistance of K. Brüggemann, M. Dehli and F. Gröschl. München: Hanser.

Dassault Systèmes (2021). Dymola®. Vélizy-Villacoublay, France. URL: https://www.3ds.com/productsservices/catia/products/dymola/ (visited on 2021-03-24).

Hamburg University of Technology (2020). TransiEnt Library. Hamburg. URL: https://www.tuhh.de/transient-ee/en (visited on 2021-03-24).

Hamburg University of Technology, TLK-Thermo GmbH, and XRG Simulation GmbH (2021). ClaRa. Hamburg, Braunschweig. URL: https://claralib.com/index.php?lang=en (visited on 2021-03-24).

Heckel, Jan-Peter, and Christian Becker (2019). "Advanced Modeling of Electric Components in Integrated Energy Systems with the TransiEnt Library". In: Proceedings of the 13th International Modelica Conference, 759-68. Linköping Electronic Conference Proceedings: Linköping University Electronic Press. DOI: 10.3384/ecp19157759.

Huber, Marcia, Allan Harvey, Eric Lemmon, Gary Hardin, Ian Bell, and Mark McLinden (2018). "NIST Reference Fluid Thermodynamic and Transport Properties Database (REFPROP) Version 10 - SRD 23". DOI: 10.18434/T4/1502528.

Klimiuk, Ewa, Zygmunt Mariusz Gusiatin, Tomasz Pokój, and Sabina Rynkowska (2015). "ADM1-Based Modeling of Anaerobic Codigestion of Maize Silage and Cattle Manure a Feedstock Characterisation for Model Implementation (Part I) / Modelowanie Kofermentacji Kiszonki Kukurydzy I Obornika Bydlęcego Za Pomocą ADM1 - Charakterystyka Wsadu Surowcowego (Część I)". Archives of Environmental Protection 41 (3): 11-19. DOI: 10.1515/aep-2015-0026.

Linkov, Igor, and José Manuel Palma-Oliveira, eds. (2017). Resilience and Risk: Methods and Application in Environment, Cyber and Social Domains. NATO science for peace and security series. Series C, Environmental security. Dordrecht: Springer Netherlands.

Modelica Association (2017-04). Modelica - a Unified ObjectOriented Language for Systems Modeling. Language Specification Version 3.4: Tech. rep. Linköping: Modelica Association. URL: https : / / www. modelica . org / documents / ModelicaSpec34.pdf.

Modelica Association (2020). Modelica ${ }^{\circledR}$ Standard Library. Linköping, Sweden: Tech. rep. Linköping: Modelica Association.

Park, R. H. (1929). "Two-Reaction Theory of Synchronous Machines Generalized Method of Analysis-Part I".
Transactions of the American Institute of Electrical

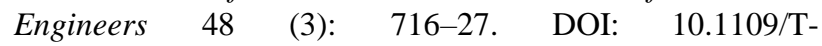
AIEE.1929.5055275.

Price, W. W., H. D. Chiang, H. K. Clark, C. Concordia, D. C. Lee, J. C. Hsu, S. Ihara et al. (1993). "Load Representation for Dynamic Performance Analysis (Of Power Systems)". IEEE Trans. Power Syst. 8 (2): 472-82. DOI: 10.1109/59.260837.

Senkel, Anne, Carsten Bode, and Gerhard Schmitz (2019). "Evaluating the Resilience of Energy Supply Systems at the Example of a Single Family Dwelling Heating System". In: Proceedings of the 13th International Modelica Conference, 655-62. Linköping: Linköping University Electronic Press. DOI: $10.3384 /$ ecp19157655.

Senkel, Anne, Carsten Bode, and Gerhard Schmitz (2021). "Quantification of the Resilience of Integrated Energy Systems Using Dynamic Simulation". Reliability Engineering \& System Safety 209:107447. DOI: 10.1016/j.ress.2021.107447.

TLK-Thermo GmbH, and Institut für Thermodynamik, Technische Universität Braunschweig (2021). "TILMedia Suite". URL: https://www.tlkthermo.com/index.php/en/software/tilmedia-suite (visited on 2021-03-16).

Webster, John, and Carsten Bode (2019). "Implementation of a Non-Discretized Multiphysics PEM Electrolyzer Model in Modelica". In: Proceedings of the 13th International Modelica Conference, 833-40. Linköping Electronic Conference Proceedings: Linköping University Electronic Press. DOI: 10.3384/ecp19157833.

Wetter, Michael, Wangda Zuo, Thierry S. Nouidui, and Xiufeng Pang (2014). Modelica Buildings Library. URL: https://simulationresearch.lbl.gov/modelica/download.html (visited on 2021-03-24). 